\title{
Farewell to paper
}

\author{
Grae Worster
}

I am sure that the news that this volume of the Journal of Fluid Mechanics (JFM) is the last to be produced in print will receive mixed reactions. Many, like myself, still prefer to read from paper than on a screen. Yet, I have to admit that it is several years since I visited a library and, even though I have ready access to volumes of JFM going back to 1994 on my bookshelves, more often than not I look for papers on the internet. I usually browse the abstracts on screen and then print off those papers I want to read. Indeed, thinking back to my pre-internet days as a $\mathrm{PhD}$ student, this practice is not very different from then: I would browse journals in the library but then photocopy the papers I was interested in because I always read with pen in hand. So, for me, the bound volume or the PDF online is principally a vehicle for delivery. Though I will join those who mourn the passing of the paper volume, I realise that this has more to do with nostalgia than practical reality.

There is, however, a serious concern related to my nostalgia. The strongest memories of my early years of research relate to the delight of handling and reading dusty volumes of the Proceedings of the Royal Society and other learned journals from the nineteenth century with papers by the likes of Lord Rayleigh or Stokes. The challenge for our scientific and other academic communities will be the maintenance of electronic forms of communication, with software changing rather more quickly than the evolution from copper-plate handwriting to standardised print fonts, the more ancient of which we can still read directly without too much difficulty.

Several factors have prompted this transition to online-only publication. The practice of scientific publishing and reading has changed enormously since JFM was founded in 1956. The early issues of JFM were produced every other month, and the first ever contained 6 papers spanning 128 pages. Current issues are produced twice a month, and this volume contains 40 papers spanning 1157 printed pages plus a 14 page online-only JFM Rapids paper. Altogether, JFM has published more than 26000 pages this year. Back then, issues were eagerly awaited and read in their entirety. Today, researchers more often scan emailed contents lists or use search engines to discover papers relevant to their particular interests. I wonder how the ratio of pages printed to pages read has changed over the years? It may not have decreased, because the community has definitely grown and so there are more people doing the reading. Nevertheless, each reader is reading an ever decreasing fraction of the total output, and the cost and environmental impact of producing and distributing large, heavy paper editions are causes for concern.

Since JFM first became available online in 1997, the Press has been maintaining two forms of publication (physical and electronic) in parallel, adding complexity to production. By introducing JFM online-only, the Press will be able to focus on its digital product and bring forward innovations that do not translate into print. Examples include inline videos and embedded code that will allow data to be viewed and analysed by readers in different ways. This is especially valuable for fluid dynamics, in which temporal evolution and structure in the third spatial 
dimension play fundamental and crucial roles. With modern experimental techniques and fast computers, the subject is now well beyond the constraints of having to study stationary, circular cows! New, imaginative ways of communicating the important scientific ideas that are now being discovered and developed are needed, and the hope is that that communication will be enhanced by digital features that cannot be replicated in print or will be lost in translation.

The look and feel of JFM is changing but the Journal is not changing in fundamentals. The hallmarks of JFM are not to be found in the weight of the printed volume but in the quality of reviewing and the quality of communication. The former ensures the very high standards of scientific development enshrined in the Journal, for which we owe huge gratitude to the community that holds the Journal in such high esteem that it is important to them to put effort into scrutinising and critiquing submissions constructively. The latter starts with the clarity of exposition expected of our authors and ends with the quality of production provided by Cambridge University Press. This seems an appropriate moment to thank and acknowledge the dedication, care and professionalism of the copy editors and typesetters that make JFM stand out amongst its peers. These qualities are not simply aesthetic but significantly reduce ambiguity and enhance scientific understanding.

So, I shall keep my volumes of JFM to gather dust on my shelves and may pull one down from time to time to reminisce. But I welcome the arrival of JFM onlineonly in 2020 and look forward to the future development of fluid mechanics being promulgated by the Journal in exciting new ways built on the substantial archive of ideas that have stood the test of time.

Grae Worster

The Journal of Fluid Mechanics will be published online-only from January 2020 with free colour for everyone, new online cover designs, the introduction of graphical abstracts and a new layout for the table of contents. In addition, each article will receive a unique electronic article number removing the need for sequential pagination in each volume. 